$\mathbb{P}$ periodica polytechnica

Civil Engineering

$58 / 3(2014) 293,299$

doi: $10.3311 /$ PPci.7523

http://periodicapolytechnica.org/ci

Creative Commons Attribution (1)

RESEARCH ARTICLE

\section{Random structural dynamic response analysis under random excitation}

\author{
Liao Jun / Xu Dafu / Jiang Bingyan
}

Received 2014-05-15, revised 2014-06-20, accepted 2014-07-07

\begin{abstract}
A numerical procedure to compute the mean and covariance matrix of the random response of stochastic structures modeled by FE models is presented. With Gegenbauer polynomial approximation, the calculation of dynamic response of random parameter system is transformed into an equivalent certainty expansion order system's response calculation. Non- stationary, non-white, non-zero mean, Gaussian distributed excitation is represented by the well known Karhunen-Loeve (K-L) expansion. The Precise Integration Method is employed to obtain the K-L decomposition of the non-stationary filtered white noise random excitation. A accurate result is obtained by small amount of K-L vectors with the vector characteristic of energy concentration, especially for the small band-width excitation. Correctness of the method is verified by the simulations. The effects to the response mean square value by different probability density functions of random parameters with the same variable coefficient are studied, and a conclusion is drawn that it is inappropriate to approximate other types of probability distribution by normal distribution.
\end{abstract}

\section{Keywords}

stochastic structures $\cdot$ dynamic response $\cdot$ precise time integration method $\cdot$ orthogonal decomposition $\cdot K-L$ decomposition $\cdot$ random vibration

\section{Liao Jun}

College of Mechanical and Electrical Engineering, Central South University, Changsha, 410001, China

e-mail: liaojun@csu.edu.cn

Xu Dafu

Aerospace System Engineering Shanghai, Shanghai, 201108, China

\section{Jiang Bingyan}

School of Aeronautics and Astronautics, Central South University, Changsha, 410001, China

\section{Introduction}

In practical engineering problems, not only the external excitations such as wind loading, seismic waves etc., demonstrate uncertainty, but also the structural parameters exhibit uncertainty. The uncertainty of structural parameters may have a strong influence on structural reliability. [1, 2], therefore it is more reasonable to consider the variability of structural physical parameters in dynamic response analysis of structures.

The double random vibration analysis, i.e. the random vibration analysis of stochastic parameter structures subjected to random excitation, attracts much interest in researchers. In current literatures there are mainly three ways to tackle this problem. The first is Monte Carlo simulation method [1]. This method is robust and powerful in this aspect of structural analysis, but it is very time-consuming. The second is stochastic finite element method. Although the random eigen value problem, static analysis problem and structural stability problem, etc. can be solved efficiently with this method [2], the stochastic finite method is haunted by the notorious secular term in random dynamic response analysis of structures. The third is orthogonal series expansion method [3-6] in which the structural response is expanded as a set of orthogonal series and the corresponding numerical characteristics are given as analytical solution. Li [6] developed an order expanded system method by applying sequential orthogonal expansion to deal with double random vibration analysis. Some numerical examples indicate that orthogonal series expansion method avoids the secular term of perturbation methods and does not have to assume the variability of structural parameters to be small. However, the method is still very timeconsuming for large degree of freedom FE model. C.A. Schenk and H.J. Pradlwarter [7. $\mid$ 9] developed a method to deal with dynamic stochastic response of FE models under non-stationary random excitation, non-zero mean, non-white, non-stationary Gaussian distributed excitation is represented by the well known $\mathrm{K}-\mathrm{L}$ expansion. For the case where the stochastic loading is described by a finite set of deterministic K-L vectors, which in fact is considered in this paper, and to deal with double random vibration analysis. Then, the classic vibration analysis method can apply to the order-expanded equation, and the probabilistic in- 
formation of double random vibration problem can be obtained.

\section{Method of analysis}

2.1 Orthogonal polynomial expansion of double random vibration system

The dynamic equilibrium equation of stochastic structural system with $n$ degrees of freedom can be written as [10]

$$
\mathbf{M} \ddot{\mathbf{X}}+\mathbf{C X}+\mathbf{K X}=\mathbf{P}(t)
$$

In which $\mathbf{M}, \mathbf{C}$ and $\mathbf{K}$ are the values of mass, damping and stiffness matrices, respectively. $\mathbf{P}(t)$ is a $n \times 1$ random excitation vector. $\ddot{\mathbf{X}}, \dot{\mathbf{X}}, \mathbf{X}$ are $n \times 1$ random acceleration, velocity and displacement vectors, respectively, whose uncertainty arise from both stochastic structural parameters and external excitation.

$$
\begin{array}{r}
\mathbf{M}=\mathbf{M}_{0}+\sum_{j=1}^{N m} \xi_{j} \mathbf{M}_{j} \\
\mathbf{C}=\mathbf{C}_{0}+\sum_{j=1}^{N c} \xi_{j} \mathbf{C}_{j} \\
\mathbf{K}=\mathbf{K}_{0}+\sum_{j=1}^{N k} \xi_{j} \mathbf{K}_{j}
\end{array}
$$

In which $\mathbf{M}_{\mathbf{0}}, \mathbf{C}_{\mathbf{0}}$ and $\mathbf{K}_{\mathbf{0}}$ are the expected value of mass, damping and stiffness matrices, respectively. $\mathbf{M}_{\mathbf{j}}, \mathbf{C}_{\mathbf{j}}$ and $\mathbf{K}_{\mathbf{j}}$ are the deviatoric components of mass, damping and stiffness matrices. $\xi_{j}$ denotes the random variable. $N_{m}, N_{k}, N_{c}$ are the number of the random variables in mass, damping and stiffness matrices, respectively.

In most of the practical engineering problems, it is rational to assume that the variability of external excitation and the variability of stochastic structural parameters are statistically independent of each other. According to the orthogonal polynomial expansion method, the response vectors in Eq. (1) can be decomposed in probability sub-space spanned by random variables $\xi_{j}$ as follows [10, 11$]$

$$
\begin{aligned}
& \mathbf{X}(\xi, \varsigma, t)= \\
& =\sum_{l_{1}=0}^{N_{1}} \sum_{l_{2}=0}^{N_{2}} \ldots \sum_{l_{R}=0}^{N_{R}} \mathbf{Y}_{l_{1}, l_{2} \ldots l_{R}}(\varsigma, t) H_{l_{1}}\left(\xi_{1}\right) H_{l_{2}}\left(\xi_{2}\right) \ldots H_{l_{R}}\left(\xi_{R}\right)
\end{aligned}
$$

$$
\begin{aligned}
& \dot{\mathbf{X}}(\xi, \varsigma, t)= \\
& =\sum_{l_{1}=0}^{N_{1}} \sum_{l_{2}=0}^{N_{2}} \ldots \sum_{l_{R}=0}^{N_{R}} \dot{\mathbf{Y}}_{l_{1}, l_{2} \ldots l_{R}}(\varsigma, t) H_{l_{1}}\left(\xi_{1}\right) H_{l_{2}}\left(\xi_{2}\right) \ldots H_{l_{R}}\left(\xi_{R}\right)
\end{aligned}
$$

$$
\begin{aligned}
& \ddot{\mathbf{X}}(\xi, \varsigma, t)= \\
& =\sum_{l_{1}=0}^{N_{1}} \sum_{l_{2}=0}^{N_{2}} \ldots \sum_{l_{R}=0}^{N_{R}} \ddot{\mathbf{Y}}_{l_{1}, l_{2} \ldots l_{R}}(\varsigma, t) H_{l_{1}}\left(\xi_{1}\right) H_{l_{2}}\left(\xi_{2}\right) \ldots H_{l_{R}}\left(\xi_{R}\right)
\end{aligned}
$$

In which, $\mathbf{Y}_{\mathbf{l}_{1} \mathbf{l}_{2} \ldots \mathbf{l}_{\mathbf{R}}}(\varsigma, t), \dot{\mathbf{Y}}_{\mathbf{l}_{1} \mathbf{l}_{2} \ldots \mathbf{I}_{\mathbf{R}}}(\varsigma, t)$ and $\ddot{\mathbf{Y}}_{\mathbf{l}_{1} \mathbf{l}_{2} \ldots \mathbf{I}_{\mathbf{R}}}(\varsigma, t)$ are $n \times 1$ unknown random processes. $\xi$ is random vector composed of the independed random variable $\xi_{j} . \varsigma$ is the random vector denotes the uncertainty of external excitation which is composed of $\theta_{j}\left(j=1,2 \ldots N_{a}\right)$ and explained in further detail below. $R$ is the number of random variables of $\xi_{j} . H_{l_{j}}\left(\xi_{j}\right)$ is a set of truncated orthogonal polynomials, whose types vary with the probability density function of stochastic structural parameters. Generally, Hermite polynomials are selected for Gaussian random variables, Legendre polynomials for uniform random variables, Laguerre polynomials for exponential random variables, Gegenbauer polynomials for $\lambda$-PDF random variables [12] etc. The most important properties of the orthogonal polynomials are their recurrence relationship and the orthogonality relationship in inner product space defined by the expectation operation with respect to random variables $\xi_{j}$. The truncation number of polynomials for every random varialbe is $N_{s}(\mathrm{~s}=1,2, \ldots, R)$, the sum of combination of the polynomials is $M_{R}=\prod_{s=1}^{R}\left(N_{s}\right)$. The order-expanded equations can be obtained by assembling these equations of sum of polynomials, and its dimension is $N=M_{R} \times n[6$

$$
\mathbf{A}_{M} \ddot{\mathbf{Y}}+\mathbf{A}_{C} \dot{\mathbf{Y}}+\mathbf{A}_{K} \mathbf{Y}=F(t)
$$

Where,

$$
\begin{gathered}
\mathbf{Y}=\left[\mathbf{Y}_{0 \ldots 0}^{T}, \mathbf{Y}_{0 \ldots 1}^{T}, \ldots, \mathbf{Y}_{0 \ldots N_{R}}^{T}, \mathbf{Y}_{0 \ldots 10}^{T}\right. \\
\left.\mathbf{Y}_{0 \ldots 11}^{T}, \ldots, \mathbf{Y}_{0 \ldots 1 N_{R}}^{T}, \ldots, \mathbf{Y}_{N_{1} N_{2} \ldots N_{R}}^{T}\right]^{T}
\end{gathered}
$$

$\mathbf{A}_{M}, \mathbf{A}_{C}$ and $\mathbf{A}_{K}$ are $N \times N$ deterministic order-expanded mass, damping and stiffness matrices, respectively. $\ddot{\mathbf{Y}}, \dot{\mathbf{Y}}$ and $\mathbf{Y}$ are unknown generalized response vectors with randomness derived from external excitation $\mathbf{F}(t)$, which is an order-expanded random load vector composed of the vectors

$$
\mathbf{F}(t)=\left[\mathbf{P}(t)^{T}, 0^{T}, \ldots, 0^{T}\right]^{T}
$$

In which the superscript " $T$ " represents the transposition of a matrix. Except for the first n-dimension vector, the other components of the generalized load vector are zero. The dynamic response of Eq. (8) can be solved by means of any classic vibration analysis methods, for the order-expanded matrices are deterministic. Because the order of the equation has been expanded, the method to reduce the computation load becomes a key problem to solve the equation, especially for double random vibration analysis.

\subsection{Modeling of stochastic loading}

The components of the vector of acceleration $a(t)$ are frequently modeled as statistically independent stochastic processes defined as filtered white noise [8]. The force vector $\mathbf{P}(t)$ then can be define as

$$
\mathbf{P}(t)=-\mathbf{M}_{0} \mathbf{I}_{a} a(t)
$$


Where $\mathbf{I}_{a}$ is a displacement transformation matrix

$$
a(t)=\mathbf{Q}_{f} \mathbf{v}(t)
$$

Where $\mathbf{Q}_{f}$ denotes a constant matrix , the filter follows the differential equation

$$
\dot{\mathbf{v}}(t)=\mathbf{A}_{f} \mathbf{v}(t)+\mathbf{b}_{f}(t) \omega(t)
$$

In which,v, $\mathbf{A}_{f}$ and $\mathbf{b}_{f}$ denote the state vector, the system matrix and the distribution matrix of the filter. $\omega(t)$ denotes the white noise random process defined as follows

$$
E\left(\omega(t) \omega^{T}(t+\tau)\right)=2 \pi \mathbf{S}_{0}(t) \delta(\tau)
$$

Where $\delta$ is the Dirac's delta function, and $\mathbf{S}_{0}(t)=\mathbf{S}_{0}$ is the constant spectral density. Then, the covariance matrix $\boldsymbol{\Gamma}_{v v}(t)$ can be obtained by the Lyapunov matrix differential equation

$$
\dot{\boldsymbol{\Gamma}}_{v v}(t)=\mathbf{A}_{f} \boldsymbol{\Gamma}_{v v}(t)+\boldsymbol{\Gamma}_{v v}(t) \mathbf{A}_{f}^{T}+\mathbf{b}_{f}(t) \mathbf{I}_{f}^{T}(t)
$$

The covariance matrix $\boldsymbol{\Gamma}_{v v}\left(t_{1}, t_{2}\right)$ can be obtained by [9]

$$
\frac{\partial}{\partial t_{1}} \boldsymbol{\Gamma}_{v v}\left(t_{1}, t_{2}\right)=\mathbf{A}_{f} \boldsymbol{\Gamma}_{v v}\left(t_{1}, t_{2}\right)
$$

In which $\boldsymbol{\Gamma}_{v v}\left(t_{2}, t_{2}\right)=\boldsymbol{\Gamma}_{v v}\left(t_{2}\right)$. Then, the covariance matrix $\boldsymbol{\Gamma}_{a a}$ can be calculated by

$$
\boldsymbol{\Gamma}_{a a}\left(t_{1}, t_{2}\right)=E\left[\mathbf{Q}_{f v}\left(t_{1}\right) \mathbf{v}^{T}\left(t_{2}\right) \mathbf{Q}_{f}^{T}\right]
$$

Precise integration [13] iterative schemes can be derived to obtain $\boldsymbol{\Gamma}_{v v}\left(t_{1}, t_{2}\right)$. Because $\mathbf{A}_{f}$ is a time-invariant matrix, its general solution can be given as

$$
\boldsymbol{\Gamma}_{v v}\left(\Delta t+t_{0}, t_{0}\right)=\exp \left(\mathbf{A}_{f} \Delta t\right) \boldsymbol{\Gamma}_{v v}\left(t_{0}\right)
$$

In which, $\boldsymbol{\Gamma}_{v v}\left(t_{0}\right)$ can be obtained by solving of Eq. 15. The transfer matrix $\mathbf{T}$

$$
\mathbf{T}=\exp \left(\mathbf{A}_{f} \Delta t\right)
$$

In which, $\Delta t$ is the time step size, and set a extremely small time interval $\tau$

$$
\tau=\Delta t / m
$$

Where, $m$ is an arbitrary integer. It is suggested to select $m=2^{N}$, in this paper, $N=20, m=1048576$. Because $\tau$ is extremely small time interval, its transfer matrix can be obtained by truncated Taylor expansion with high precision.

$$
\exp \left(\mathbf{A}_{f} \tau\right) \approx I+\left(\mathbf{A}_{f} \tau\right)+\frac{\left(\mathbf{A}_{f} \tau\right)^{2}}{2}+\frac{\left(\mathbf{A}_{f} \tau\right)^{3}}{3 !}
$$

Let $\mathbf{T}_{a}$ equal

$$
\mathbf{T}_{a}=\left(\mathbf{A}_{f} \tau\right)+\frac{\left(\mathbf{A}_{f} \tau\right)^{2}}{2}+\frac{\left(\mathbf{A}_{f} \tau\right)^{3}}{3 !}
$$

The follow instruction is executed for $N=20$ times

$$
\mathbf{T}_{\mathbf{a}}=2 \mathbf{T}_{a}+\mathbf{T}_{a} \times \mathbf{T}_{a}
$$

T can be obtained by the summation

$$
\mathbf{T}=\mathbf{I}+\mathbf{T}_{a}
$$

$\boldsymbol{\Gamma}_{v v}\left(t_{1}, t_{2}\right)$ can be obtained by the follow equation and its symmetry characteristic:

$$
\boldsymbol{\Gamma}_{v v}\left(t_{2}+(k+1) \cdot \Delta t, t_{2}\right)=\mathbf{T} \cdot \boldsymbol{\Gamma}_{v v}\left(t_{2}+k \cdot \Delta t, t_{2}\right)
$$

$\boldsymbol{\Gamma}_{a a}\left(t_{1}, t_{2}\right)$ can be obtained by Eq. (17).

The K_L expansion [14] is quite often used for modelling of stochastic loading process in recent years. A discrete, Gaussian, second-order stochastic process $\mathbf{A}_{a}$ can be obtained after discretization of the corresponding continuous stochastic process $a(t)$ with respect to time.

$$
\mathbf{A}_{a}=\left(a\left(t_{1}\right), \ldots, a\left(t_{N_{a}}\right)\right)^{T}
$$

Where, $N_{a}$ denote the number of discrete time. Its K_L expansion

$$
\mathbf{A}_{a}=\boldsymbol{\mu}_{a}+\sum_{j=1}^{N_{a}} \theta_{j} \sqrt{\lambda_{j}} \boldsymbol{\psi}_{j} \equiv \mathbf{A}_{a}^{(0)}+\sum_{j=1}^{N_{a}} \theta_{j} \mathbf{A}_{a}^{(j)}
$$

Where $\boldsymbol{\mu}_{a}$ denote the mean vector of $\mathbf{A}_{a}$. The K_L vectors $\mathbf{A}_{a}^{(j)}$ are determined by solving the algebraic eigen problem. $\mathbf{A}_{a}^{(0)}$ denote the mean function.

$$
\boldsymbol{\Gamma}_{A} \boldsymbol{\psi}_{j}=\lambda_{j} \boldsymbol{\psi}_{j}
$$

$$
\mathbf{A}_{a}^{(j)}=\sqrt{\lambda_{j}} \psi_{j}
$$

Where $\boldsymbol{\Gamma}_{A}$ is the covariance matrix of the discrete random process $\mathbf{A}_{a}$, and can be obtained by Eq. (16) after discretization the corresponding continuous stochastic process $a(t)$ with respect to time. The Gaussian random variable $\theta_{j}$ is defined as

$$
\theta_{j}=\frac{1}{\sqrt{\lambda_{j}}} \mathbf{A}_{a}^{T} \boldsymbol{\psi}_{j}
$$

The eigenvalues $\lambda_{j}$ decrease rather quickly with the increasing of $j$ [9], and this characteristic is used in this paper to reduce the computation workload by truncation

$$
\tilde{\mathbf{A}}_{a} \approx \boldsymbol{\mu}_{a}+\sum_{j=1}^{m_{a}} \theta_{j} \sqrt{\lambda_{j}} \psi_{j} \quad\left(m_{a}<N_{a}\right)
$$

The covariance of $\mathbf{A}_{a}$ in any two discrete time $t_{1}$ and $t_{2}$ can be given by

$$
\boldsymbol{\Gamma}_{A}\left(t_{1}, t_{2}\right)=\sum_{j=1}^{N_{a}} \mathbf{A}_{a}^{(j)}\left(t_{1}\right) \mathbf{A}_{a}^{(j)}\left(t_{2}\right)
$$


In particular, the variance can be obtain by

$$
\boldsymbol{\Gamma}_{A}\left(t_{1}\right)=\sigma_{A}^{2}\left(t_{1}\right)=\sum_{j=1}^{N_{a}} \mathbf{A}_{a}^{(j)^{2}}\left(t_{1}\right)
$$

The absolute mean square error by truncation is

$$
\varepsilon=E\left[\left(\tilde{\mathbf{A}}_{a}-\mathbf{A}_{a}\right)^{2}\right]=E\left[\left(\sum_{j=m_{a}+1}^{N_{a}} \mathbf{A}_{a}^{(j)}\right)^{2}\right]=\sum_{j=m_{a}+1}^{N_{a}} \lambda_{j}
$$

The mean square relative error is

$$
\sigma=\frac{\varepsilon}{E\left[\mathbf{A}_{a}^{2}\right]}=\frac{\sum_{j=m_{a}+1}^{N_{a}} \lambda_{j}}{\mu_{a}^{2}+\sum_{j=m_{a}+1}^{N_{a}} \lambda_{j}}
$$

In this paper, the loading process is a non-stationary, Gaussian filtered noise process. Its power spectral density $G_{a a}(\omega)$, corresponding to the revised Kanai-Tajimi filter described, approaches to zero for $\omega \rightarrow 0$. This behaviour describes an earthquake acceleration in more realistic way than traditionally used Kanai-Tajimi filter, because for the latter one finite displacements do exist after the excitation process has vanished, see e.g. [15]. In Eqs. [12, 13, it is assumed that

$$
\begin{gathered}
\mathbf{Q}_{\mathbf{f}}=\left[\begin{array}{llll}
\Omega_{1 g}^{2} & 0 & -\Omega_{2 g}^{2} & -2 \zeta_{2 g} \Omega_{2 g}
\end{array}\right] \\
\mathbf{A}_{f}=\left[\begin{array}{cccc}
0 & 1 & 0 & 0 \\
-\Omega_{1 g}^{2} & -2 \zeta_{1 g} \Omega_{1 g} & 0 & 0 \\
0 & 0 & 0 & 1 \\
\Omega_{1 g}^{2} & 2 \zeta_{1 g} \Omega_{1 g} & -\Omega_{2 g}^{2} & -2 \xi_{2 g} \Omega_{2 g}
\end{array}\right] \\
\mathbf{b}_{f}=\left[\begin{array}{c}
0 \\
e(t) \\
0 \\
0
\end{array}\right]
\end{gathered}
$$

Where the time modulation function $e(t)$ is assumed to be

$$
e(t)=\frac{e^{-a t}-e^{-b t}}{\max \left(e^{-a t}-e^{-b t}\right)}
$$

A time span of $0,20 \mathrm{~s}$ is integrated with a time step $\Delta t=0.01 \mathrm{~s}$ yielding a size $2001 \times 1$ for discrete stochastic process $\mathbf{A}_{a}$ and $2001 \times 2001$ for $\boldsymbol{\Gamma}_{A}$. In Eqs. (33)- 36), the values $\Omega_{1 g}=15 \mathrm{rad} / \mathrm{s}, \zeta_{1 g}=0.8, \Omega_{2 g}=0.3 \mathrm{rad} / \mathrm{s}, \zeta_{2 g}=0.995$, and the white noise intensity $2 \pi \mathbf{S}_{\mathbf{0}}=0.18 \mathrm{~m}^{2} / \mathrm{s}^{3}$ are used for the filter system matrix. The first 1000 eigenvalues of covariance matrix $\boldsymbol{\Gamma}_{A}$ are shown in Fig. 1 in a semi-logarithmic (base 10) scale, and the eigenvectors $\boldsymbol{\Psi}_{\mathbf{j}}(t)(j=50,100,200)$ of the covariance matrix $\boldsymbol{\Gamma}_{A}$ are shown in Fig. 2 .

It can be seen from Fig. 1, the eigenvalues $\lambda_{j}$ decrease very quickly for increasing index $j$. The lower order eigenvectors are acting localized, too. For $\mathbf{K} \_\mathbf{L}$ vectors, their contribution to the overall response is negligible and could be omitted with increasing index $j$. This would increase the computational efficiency of the proposed method by truncation of $\mathbf{K} \_\mathbf{L}$ vectors.

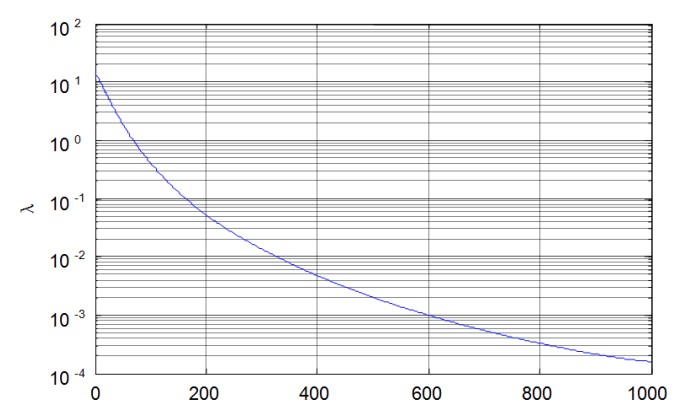

Fig. 1. The first 1000 Eigenvalues of $\boldsymbol{\Gamma}_{A}$

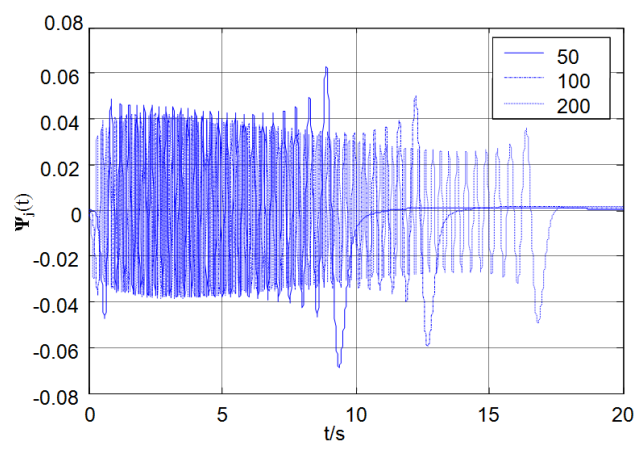

Fig. 2. Eigenvectors $\boldsymbol{\Psi}_{j}(t)$ of the covariance matrix $\boldsymbol{\Gamma}_{A}(j=50,100,200)$

\section{Dynamic response analysis of random structures}

\subsection{Method for response analysis}

The external force loading vector in this paper, is presented by the $\mathrm{K}-\mathrm{L}$ representation as stated above. In accordance to Eqs. (8) - 10,

$$
\begin{aligned}
& \mathbf{P}(t) \approx \mathbf{P}^{(0)}(t)+\sum_{j=1}^{m_{a}} \theta_{j} \mathbf{P}^{(j)}(t) \equiv \\
& \equiv-\mathbf{M}_{0} \mathbf{I}_{a}\left(\mathbf{A}_{a}^{(0)}(t)+\sum_{j=1}^{m_{a}} \theta_{j} \mathbf{A}_{a}^{(j)}(t)\right)
\end{aligned}
$$

Where, $\mathbf{P}^{(0)}(t)$ and $\mathbf{P}^{(j)}(t)$ denote the mean function and the $j$-th K - L vector of random force $\mathbf{P}(t)$, respectively. $\mathbf{I}_{a}$ is a displacement transformation matrix [9]. $\mathbf{M}_{0}$ is the structural mass matrix defined in Eq. 48. $m_{a}$ denotes the truncated quantity of $\mathrm{K}$ - $\mathrm{L}$ vectors, and it depends on the accuracy requirement referring to the error Eqs. (33) - 34.

According to linear superposition principle, the response must have the same form as Eq. (40] [7]

$$
\mathbf{Y}(t) \approx \mathbf{Y}^{(0)}(t)+\sum_{j=1}^{m_{a}} \theta_{j} \mathbf{Y}^{(j)}(t)
$$

Where $\mathbf{Y}^{(0)}(t)$ and $\mathbf{Y}^{(j)}(t)$ denote the mean function and the $j$-th $\mathrm{K}$ - L vector of displacement response, respectively. This can be done by solving Eq. (8) for every deterministic excitation $\mathrm{K}-\mathrm{L}$ vector $\mathbf{P}^{(j)}(t)$ with any deterministic linear structure dynamic analysis algorithm. After the $\mathrm{K}$ - $\mathrm{L}$ vectors of displacement response are obtain, the covariance matrix of displacement 
response can be obtained by

$$
\boldsymbol{\Gamma}_{Y Y}\left(t_{1}, t_{2}\right)=\sum_{j=1}^{m_{a}} \mathbf{Y}^{(j)}\left(t_{1}\right) \cdot \mathbf{Y}^{(j)}\left(t_{2}\right)
$$

In particular

$$
\boldsymbol{\Gamma}_{Y Y}\left(t_{1}\right)=\sigma_{Y}^{2}\left(t_{1}\right)=\sum_{j=1}^{m_{a}} \mathbf{Y}^{(j)^{2}}\left(t_{1}\right)
$$

The probabilitic information of original structural response can be obtained in terms of the response of order-expanded system by utilizing the orthogonality relationship of orthogonal polynomials $H_{l_{j}}\left(\xi_{j}\right)$. Taking the displacement response as an example, the expected vector is:

$$
\begin{gathered}
E[\mathbf{X}(\xi, \varsigma, t)]=E\left[\mathbf{Y}_{0 \ldots 0}^{T}(\varsigma, t)\right] \\
E\left[\mathbf{X}\left(\xi, \varsigma, t_{1}\right) \mathbf{X}\left(\xi, \varsigma, t_{2}\right)^{T}\right]= \\
=E\left[\sum_{l_{1}=0}^{N_{1}} \sum_{l_{2}=0}^{N_{2}} \ldots \sum_{l_{R}=0}^{N_{R}} \mathbf{Y}_{l_{1} l_{2} \ldots l_{R}}\left(\varsigma, t_{1}\right) \mathbf{Y}_{l_{1} l_{2} \ldots l_{R}}\left(\varsigma, t_{2}\right)^{T}\right]
\end{gathered}
$$

\subsection{DOFs shear wall}

In the following, the non-stationary stochastic response of a 3 DOFs shear wall with stochastic structural parameter, see Fig. 3 will be analysed in order to demonstrate the feasibility of the proposed procedure.

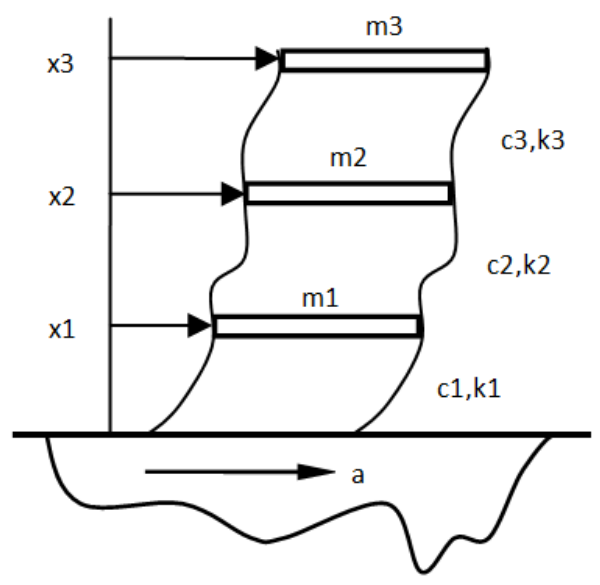

Fig. 3. 3 DOFs shear wall model

The dynamic equilibrium equation of stochastic structural system can be written as

$$
\mathbf{M} \ddot{\mathbf{x}}+\mathbf{C} \dot{\mathbf{x}}+\mathbf{K x}=-\mathbf{M u} a(t)
$$

In which, $\mathbf{M}, \mathbf{C}$ and $\mathbf{K}$ are the values of mass, damping and stiffness matrices, respectively. Considering the mass matrices has stochastic parameter.

$$
m_{3}=m_{3}^{\prime}\left(1+\mu \xi_{1}+v \xi_{1}^{2}\right)
$$

The form of $\mathbf{M}$ in Eq. 46 is

$$
\mathbf{M}=\mathbf{M}_{0}+\xi_{1} \mathbf{M}_{1}+\xi_{1}^{2} \mathbf{M}_{2}
$$

$$
\begin{gathered}
\mathbf{M}_{0}=\left[\begin{array}{ccc}
m_{1} & 0 & 0 \\
0 & m_{2} & 0 \\
0 & 0 & m_{3}^{\prime}
\end{array}\right], \\
\mathbf{M}_{1}=\left[\begin{array}{ccc}
0 & 0 & 0 \\
0 & 0 & 0 \\
0 & 0 & \mu m_{3}^{\prime}
\end{array}\right], \\
\mathbf{M}_{2}=\left[\begin{array}{ccc}
0 & 0 & 0 \\
0 & 0 & 0 \\
0 & 0 & v m_{3}^{\prime}
\end{array}\right]
\end{gathered}
$$

$$
\begin{aligned}
& \mathbf{C}=\left[\begin{array}{ccc}
c_{1}+c_{2} & -c_{2} & 0 \\
-c_{2} & c_{2}+c_{3} & -c_{3} \\
0 & -c_{3} & c_{3}
\end{array}\right], \\
& \mathbf{K}=\left[\begin{array}{ccc}
k_{1}+k_{2} & -k_{2} & 0 \\
-k_{2} & k_{2}+k_{3} & -k_{3} \\
0 & -k_{3} & k_{3}
\end{array}\right] \\
& \mathbf{u}=[1,1,1]^{T}, \quad \mathbf{x}=\left[x_{1}, x_{2}, x_{3}\right]^{T}
\end{aligned}
$$

$\xi_{1}$ denotes the $\lambda$ - PDF independent random variables as stated in [12], $\lambda=2.5$, and the variability coefficient is 0.187 . In the Gegenbauer polynomial approximation, we took $G_{0}^{\lambda}\left(\xi_{1}\right)$ up to $G_{3}^{\lambda}\left(\xi_{1}\right)$ as the orthogonal polynomial basis. Probability distribution function of $\xi_{1}$ is shown in Fig. 4 as the curve of normal distribution. $a(t)$ is fixed by Eq. (36) - 39). Other parameters are taken as: $\mu=0.5, v=0.25, m_{1}=m_{2}=m_{3}^{\prime}=1.75 \times 10^{4} \mathrm{~kg}$, $k_{1}=k_{2}=k_{3}=3.5 \times 10^{7} \mathrm{~N} / \mathrm{m}, c_{1}=c_{2}=c_{3}=1.25 \times 10^{5} \mathrm{~N} \mathrm{~s} / \mathrm{m}$.

After assembling order-expanded system as Eq. (8), the response mean square of the 3rd floor (m3) is obtained by the proposed procedure with number of $40,60,80,120 \mathrm{~K}$ - L vectors, as shown in Fig. 5. Monte Carlo random simulation method is used to verify the feasibility of the proposed procedure.

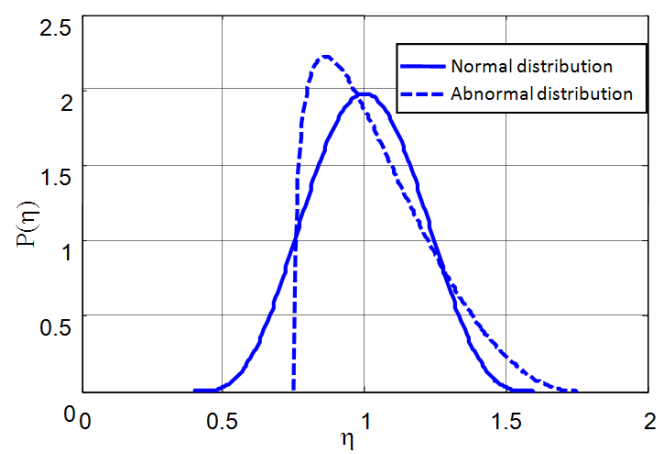

Fig. 4. Two probability density functions with the same variation coefficient

In Fig. 5, it is possible to verify the error due to truncation of the $\mathrm{K}$ - $\mathrm{L}$ expansion. The responses of $\mathrm{m} 3$ with different number of the $\mathrm{K}-\mathrm{L}$ expansion are obtained and compared with the 


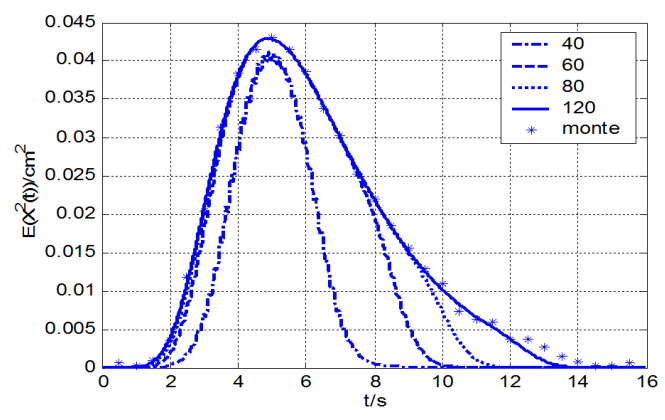

Fig. 5. The mean square curve of $m 3$ with different K_L numbers

result obtained by Monte Carlo method (1000 times). According to these results, $80 \mathrm{~K}$ - L vectors are sufficient to obtain a good result for the 3 DOFs system over the time span $[0,9] \mathrm{s}$. The maximum of the response is captured very well, and it is important for structure reliability analysis.

Mean square value calculated by the proposed method with $160 \mathrm{~K}$ _L vectors is shown in Fig. 6, and 'monte' stands for the result calculated by Monte Carlo method (1000 times). It verifies the feasibility of the proposed procedure, and the error is rather small, when the number of $\mathrm{K} \_\mathrm{L}$ vector is more than 160 .

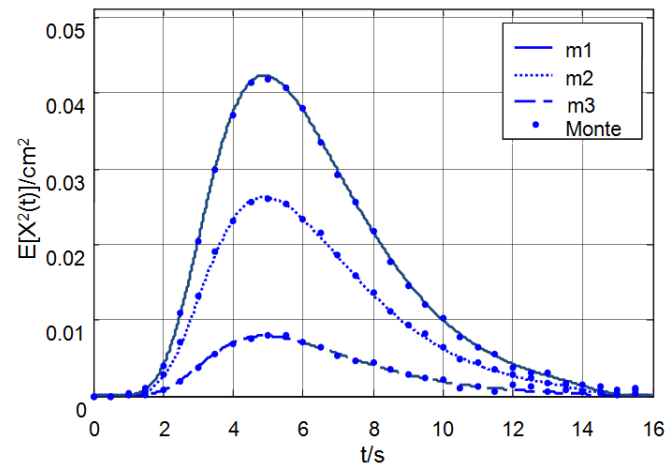

Fig. 6. Mean square value with $m_{a}=160$

In the following, another set of parameters are fixed as $\mu=0.602, v=q, 0, \lambda_{1}=4.2$. The PDF of $m_{3}$ is shown in Fig. 4 as the abnormal distribution curve, while the variability coefficient is still set as 0.187 .

The mean square values of response of the 3 floors are shown in Fig. 7. It is shown that the magnitude of deviation of the 2 results calculated by different random structural parameters are cannot be ignored, especially at the peak of the mean square values, the relative error at peak of $\mathrm{m} 3$ is up to $18 \%$. Therefore, wrong result may be obtained by treating all the random structure PDF as normal distribution in some situations.

\subsection{DOFs shear wall}

A shear wall subjected to a non-stationary seismic excitation defined by Eq. (36) - 39) is shown in Fig. 8 The size parameters are given as: width $W=1.0 \mathrm{~m}$, height $H=20.0 \mathrm{~m}$, thickness $T H=0.25 \mathrm{~m}$. The type of 8-node planar isoparametric element is selected for the 10 partitioned two-dimensional finite elements of the structure, and the sum of freedom is 106. The mean values of the structural parameters are given as: poison

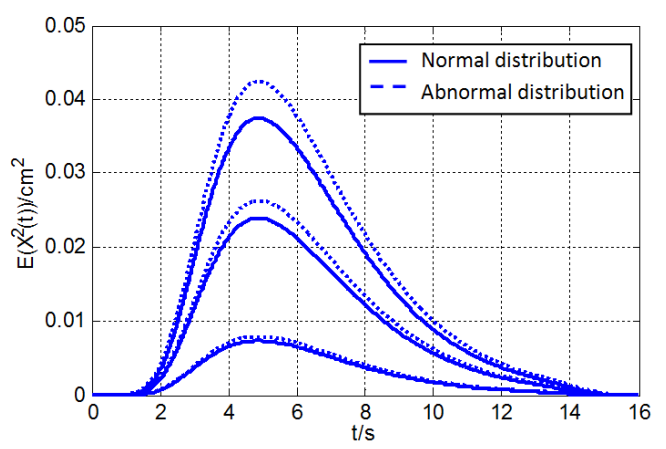

Fig. 7. Mean square values calculated with 2 different probability density functions.

ratio $\mu_{0}=0.167$, elasticity modulus $E_{0}=2.0 \times 10^{4}$, mass density $\rho_{0}=2.5 \times 10^{3} \mathrm{~kg} / \mathrm{m}^{3}$, damping ratio 0.05 . The uncertainty of the elasticity modulus $\mathrm{E}$ is described as $\lambda$-PDF random variable and the probability density functions as shown in Fig. 4 with solidline as stated above and the variability coefficient is still 0.187. A 3-order polynomial approximation is used for the random variables. Number of $10,30,50,80 \mathrm{KL}$ vectors is used to obtain the displacement variance $\sigma(t)$ of the top floor, Monte Carlo random simulation (1000 times) is employed to verify the feasibility, and 1000 times random simulation is done to get the displacement variance.

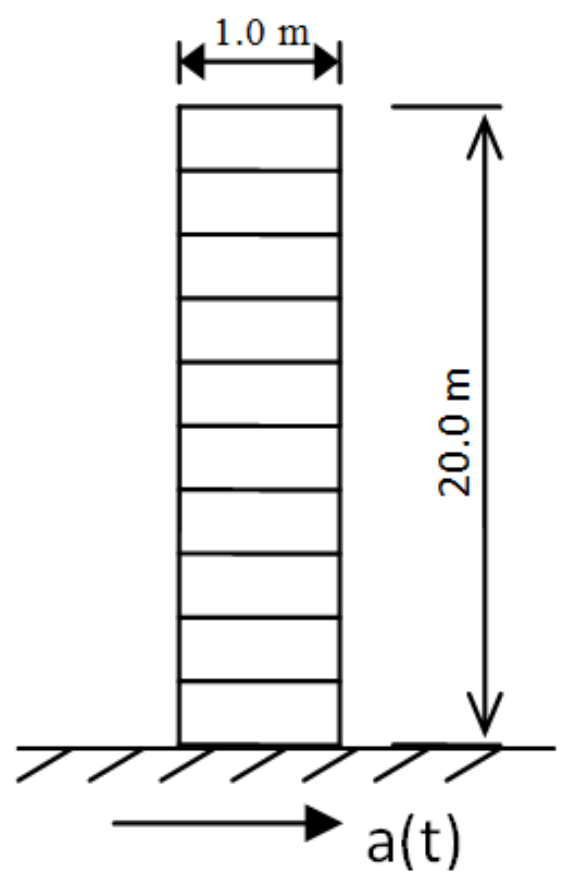

Fig. 8. The dimension and element meshes of shear wall

The result is shown in Fig. 9 According to the result, $80 \mathrm{~K}$ $\mathrm{L}$ vectors are sufficient to obtain a good result for this $9 \mathrm{DOF}$ system. The same as the above simulation, the accuracy near the peak value is good, and only $30 \mathrm{~K}$ - L vectors can get a rather good result of the peek value. It is important for structural reliability calculation. If the number of $\mathrm{K}-\mathrm{L}$ vectors less than 10 , the result is not desirable. 


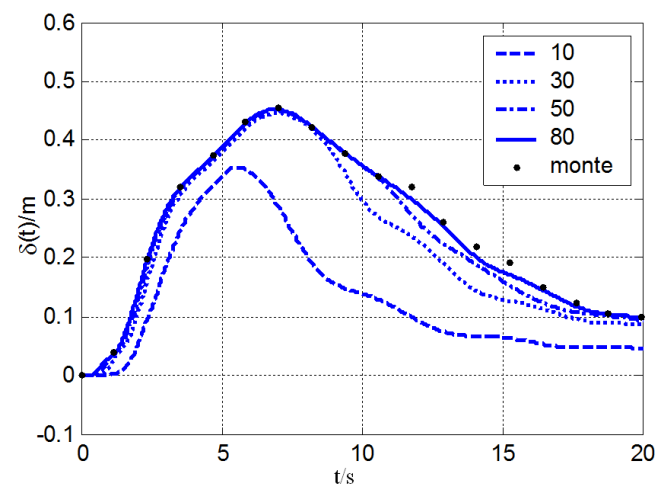

Fig. 9. Comparison of $\sigma(t)$ for different number of K_L

\section{Conclusions}

The presented development and the simulation work allow to draw the following conclusions:

- The proposed method is well adapted for the calculation of the non-stationary stochastic response of stochastic structure, and it avoids the problem of secular term appears in method of perturbation.

- Non-white, non-zero mean, non-stationary Gaussian distributed excitation is represented by the well known $\mathrm{K}-\mathrm{L}$ expansion, which allows to describe any type of non-white Gaussian excitation, and by employing K - L orthogonal decomposition and stochastic structure orthogonal decomposition, an increase in efficiency is desirable.

- The procedure allows the use of well known deterministic integration algorithm for the solution of the expanded dynamic equation; hence the application of compound stochastic analysis in the engineering practice is greatly enhanced.

\section{Acknowledgement}

This research is supported by Central South University Postdoctoral Fund.

\section{References}

1 Astill C, Imosseir S, Shinozuka M, Impact Loading on Structures with Random Properties, Journal of Structural Mechanics, 1(1), (1972), 63-77, DOI $10.1080 / 03601217208905333$

2 Contreras $\mathbf{H}$, The stochastic finite-element method, Computers \& Structures, 12(3), (1980), 341-348, DOI 10.1016/0045-7949(80)90031-0

3 Sun T, A Finite Element Method for Random Differential Equations with Random Coefficients, SIAM Journal on Numerical Analysis, 16(6), (1979), 1019-1035, DOI $10.1137 / 0716075$

4 Ghanem R, Spanos P, Polynomial Chaos in Stochastic Finite Elements, Journal of Applied Mechanics, 57(1), (1990), 197-202, DOI $10.1115 / 1.2888303$

5 Jensen H, Iwan WD, Response of Systems with Uncertain Parameters to Stochastic Excitation, Journal of Engineering Mechanics, 118(5), (1992), 1012-1025, DOI 10.1061/(ASCE)0733-9399(1992)118:5(1012)

$6 \mathbf{L i} \mathbf{J}$, The expanded order system method combined random vibration analysis, ACTA Mechanica Sinica, 28(3), (1996), 66-75.

7 Schueller GI, Pradlwarter HJ, On the stochastic response of nonlinear FE models, Archive of Applied Mechanics (Ingenieur Archiv), 69(9), (1999), 765-784.
8 Schueller GI, Pradlwarter HJ, Schenk CA, Non-stationary response of large linear FE models under stochastic loading, Computers and structures, 81(8-11), (2003), 937-947, DOI 10.1016/S0045-7949(02)00473-X

9 Schenk CA, Pradlwarter HJ, Schueller GI, On the dynamic stochastic response of FE models, Probabilistic Engineering Mechanics, 19(1-2), (2004), 161-170, DOI $10.1016 /$ j.probengmech.2003.11.013

10 Li J, Liao ST, Response analysis of stochastic parameter structures under non-stationary random excitation, Computational Mechanics, 27(1), (2001), 61-68, DOI 10.1007/s004660000214

11 Xiu D, Numerical methods for stochastic computations: a spectral method approach, Princeton University Press, 2010.

$12 \mathrm{Ma} \mathrm{X}$, Leng X, Meng G, Fang T, Evolutionary earthquake response of uncertain structure with bounded random parameter, Probabilistic Engineering Mechanics, 19(3), (2004), 239-249, DOI 10.1016/j.probengmech.2004.02.007

13 Zhong W, On precise integration method, Journal of Computational and Applied Mathematics, 163(1), (2004), 59-78, DOI 10.1016/j.cam.2003.08.053

14 Ghanem RG, Spanos PD, Stochastic finite elements: a spectral approach, Courier Dover Publications, 2003.

15 Humar J, Dynamics of structures, CRC Press, 2012. 